\title{
La réglementation cosmétique et ses adaptations aux matières premières cosmétiques
}

\author{
Françoise CHANTELOUBE \\ Formation et conseil en cosmétique (FCC) \\ 17, quai Georges Clémenceau \\ 78380 Bougival \\ $<$ francoise.chanteloube@wanadoo.fr>
}

\begin{abstract}
Cosmetic industry has since many years (1976) taken the party to frame on the legislative level the launch of cosmetic products on the market. This regulation takes into account the finished product as well as the ingredients or mixtures of raw materials, which composes it. This article proposes to review the essential points which relate to the use of the raw materials in cosmetic and their specific lawful aspects.
\end{abstract}

Key words: cosmetic industry, cosmetic products, european reglementation

indication ou information émanant du fabricant ou de son mandataire ou de tout autre responsable de la mise sur le marché... »

Cet article est un point-clé de cette directive et nous permet de présager que nombre d'articles de cette législation concerneront la sécurité du consommateur final et plus particulièrement les points faisant référence aux ingrédients qui composeront ces produits.

\section{Les ingrédients cosmétiques}

L'article 4 concerne tout particulièrement les ingrédients cosmétiques et certaines de leurs modalités d'utilisation dans les formulations cosmétiques. Les annexes (tableau 1) de cet article définissent les points suivants :

- La liste négative.

L'annexe II liste les ingrédients qui ne peuvent pas entrer dans la composition de produits mis sur le marché européen. Une limitation est cependant autorisée pour des traces de ces substances qui seraient techniquement inévita- bles dans le respect des bonnes pratiques de fabrication à conditions que ces dernières ne soient pas un risque pour la santé du consommateur, comme mentionné à l'article 2.

- La liste des ingrédients limités.

L'annexe III : liste un ensemble d'ingrédients utilisables uniquement sous certaines conditions :

- des limitations de concentrations : certains ingrédients ne pourront pas être utilisés au-delà d'une concentration donnée ;

- des restrictions de zones cutanées : certains ingrédients pourront être utilisés dans des produits appliqués sur la peau et pas pour des produits appliqués sur les muqueuses ou les yeux ;

- des spécifications d'utilisateurs finaux : certains ingrédients pourront être limités voire interdits pour incorporation dans des produits cosmétiques destinés à des enfants de moins de 3 ans... ;

- des limitations quant au mode d'utilisation : certains ingrédients seront permis dans des mises en contact avec les diverses parties superficielles du corps humain (épiderme, système pileux et capillaire, ongles, lèvres et organes génitaux extérieurs) ou avec les dents et les muqueuses, en vue, exclusivement ou principalement, de les nettoyer, de les parfumer, d'en modifier l'aspect et/ou de corriger les odeurs corporelles, et/ou de les protéger ou de les maintenir en bon état. »

Cette définition mentionne le site d'application des produits et la finalité de leurs actions.

\section{Les responsabilités}

L'article 2 quant à lui fixe les responsabilités qui incombent au metteur sur le marché :

« Les produits cosmétiques mis sur le marché, ne doivent pas nuire à la santé humaine lorsqu'ils sont appliqués dans les conditions normales ou raisonnablement prévisibles d'utilisation, compte tenu notamment de la présentation du produit, de son étiquetage, des instructions éventuelles concernant son utilisation et son élimination, ainsi que de toute autre

Tableau 1. Ingrédients cosmétiques réglementés.

\begin{tabular}{|c|c|}
\hline Annexe II & $\begin{array}{l}\text { Substances qui ne peuvent entrer dans la composition des produits cosmétiques ( } 451 \\
\text { ingrédients ou familles d'ingrédients). }\end{array}$ \\
\hline Annexe III & $\begin{array}{l}\text { Substances que les produits cosmétiques ne peuvent contenir en dehors des restrictions } \\
\text { et conditions prévues ( } 95 \text { ingrédients définitivement admis et } 62 \text { admis provisoirement) : } \\
\text { champ d'application et/ou usages, concentration maximale autorisée, autres limitations } \\
\text { ou exigences, conditions d'emploi et mentions obligatoires. }\end{array}$ \\
\hline Annexe IV & $\begin{array}{l}\text { Substances colorantes autres que celles citées dans la présente annexe ( } 146 \text { ingrédients) : } \\
\text { utilisation dans tous produits cosmétiques, avec restriction pour les produits appliqués } \\
\text { près des yeux, avec restriction pour l'application sur muqueuses, utilisation uniquement } \\
\text { dans les produits rincés ou en contact bref avec la peau, colorants destinés à colorer } \\
\text { uniquement le système pileux. }\end{array}$ \\
\hline Annexe V & Substances exclues du champ d'application de la Directive : Strontium et ses sels. \\
\hline Annexe VI & $\begin{array}{l}\text { Agents conservateurs autres que ceux cités dans la présente annexe ( } 56 \text { conservateurs } \\
\text { admis) : concentration maximale, limites et exigences, conditions d'emploi et mentions } \\
\text { obligatoires. }\end{array}$ \\
\hline Annexe VII & $\begin{array}{l}\text { Filtres ultraviolets autres que ceux cités dans la présente annexe ( } 27 \text { filtres admis) : } \\
\text { concentration maximale, limites et exigences, conditions d'emploi et mentions } \\
\text { obligatoires. }\end{array}$ \\
\hline
\end{tabular}


Tableau 2. Allergènes de parfumerie.

\begin{tabular}{|ll|}
\hline 2-pentyl-3-phenylprop-2ene-1-ol & Cinnamaldéhyde \\
Salicylate de benzyle & 2-benzylideneheptanal \\
Alcool 4methoxybenzylique & Alcool benzylique \\
Coumarine & Benzoate de benzyle \\
Geraniol & Cinnamate de benzyle \\
7-Hydroxycitronellal & Alcool cinnamylique \\
4-(4-Hydroxy-4-methylpentyl) & 3-methyl-4-(2,6,6-trimethyl-2-cyclohexene-1-yl)-3butene-2- \\
cyclohex-3enecarbaldehyde & one \\
Isoeugenol & Citral \\
Octyl-2-ynoate de methyle & Citronellol \\
(R)-p-mentha-1,8-diene & Eugénol \\
2-(4-tert-butylbenzyl)propionaldehyde & Farnesol \\
Evernia prunastri, extraits & Hexyl cinnamaldehyde \\
Evernia furfuracea, extraits & Linalol \\
\hline
\end{tabular}

produits rincés qui ne restent qu'en bref contact avec la peau alors qu'ils pourront être limités ou interdits pour une incorporation dans des produits non rincés et qui restent en contact avec la peau ;

- certains ingrédients de parfumerie pouvant présenter des risques allergiques pour les consommateurs sont à mentionner dans le listing des ingrédients à des fins d'information pour ces derniers uniquement lorsque leur concentration dans le produit fini sera supérieure à 100 ppm dans des produits rincés et 10 ppm dans des produits non rincés (tableau 2). - Les listes positives.

Les annexes IV, VI et VII sont respectivement les listes positives des matières colorantes, des agents conservateurs et des filtres solaires à usage en cosmétique. Ces listes positives concernent trois familles d'ingrédients cosmétiques non dénués de risques toxicologiques et cela de façon inhérente à leur structure chimique et à leur mode d'action.

Ces listes positives fixent les règles $d^{\prime}$ usage de ces substances :

- concentrations maximales d'utilisation pour les conservateurs et les filtres solaires ;

- conditions d'utilisation des matières colorantes : Catégorie 1 : utilisation dans tous les produits cosmétiques; Catégorie 2 : restriction dans les produits appliqués près des yeux; Catégorie 3 : restriction pour l'application sur les muqueuses; Catégorie 4 : utilisation uniquement dans produits rincés ou en contact bref avec la peau ; Catégorie 5 : colorants destinés à colorer uniquement le système pileux ; - des mentions particulières à reprendre sur l'emballage des produits.

\section{L'expérimentation animale}

L'article 4bis donne la position du législateur quant à la réalisation d'expérimentation animale concernant les ingrédients cosmétiques et les produits finis mis sur le marché.
Interdiction de la mise sur le marché de produits cosmétiques dont la formule finale aura fait l'objet de tests sur animaux par une méthode autre qu'une méthode alternative, après que celle-ci ait été validée et adoptée au niveau communautaire.

Interdiction de la mise sur le marché de produits cosmétiques contenant des ingrédients ou des combinaisons d'ingrédients ayant fait I'objet d'une expérimentation animale par une méthode autre qu'une méthode alternative, après que celle-ci ait été validée et adoptée au niveau communautaire.

En absence de méthodes alternatives validées et adoptées, les dates limites sont de :

- 6 ans à compter de la transcription de la Directive ;

- 10 ans pour les tests de toxicité répétée, de reprotoxicité et toxicocinétique.

\section{Les substances CMR}

L'article 4ter rend compte des règles législatives d'utilisation des substances classées comme CMR: substances cancérogènes, mutagènes ou toxiques pour la reproduction. Ces dernières peuvent appartenir aux trois classes suivantes:

- Classe 1 : Substances que I'on sait être toxiques pour I'homme ;

- Classe 2 : Substances devant être assimilées à des toxiques pour I'homme ;

- Classe 3 : Substances préoccupantes pour I'homme.

Interdiction systématique des substances classées CMR 1 et 2 dans les produits cosmétiques. En ce qui concerne les substances classées CMR 3 , ces dernières sont interdites à I'utilisation dans les produits cosmétiques, sauf avis favorable du SCCNFP.

\section{Inventaire des ingrédients cosmétiques}

L'article 5 bis définit deux inventaires indicatifs des ingrédients : un pour les ingrédients cosmétiques et l'autre pour les ingrédients à usages parfumants et aromatiques.

Cet inventaire reprend un ensemble d'identifiants des matières premières: $N^{\circ} C A S$, IUPAC NAME, les numéros ENEICS ou EILINCS, les dénominations $I \mathrm{NCl}$...

- Le CAS, Chemical Abstract Service (Étatsunis), assure l'enregistrement mondial des substances chimiques depuis 1907. Le dossier prend en compte :

- définition des composants chimiques: diagramme de la substance chimique, nom chimique, noms communs, formule chimique et moléculaire, isoméries de position ou stéréochimiques, les formes salines, les configurations stéréochimiques, les formes physiques non naturelles...

- présence de polymères: nature des monomères, système de réaction, rôle des réactifs, structure et diagramme polymérique...

- réactions complexes : réactifs et nature des réactions, composition finale du produit ;

- traçabilité des végétaux: famille, genre, espèce, sous espèce, hybrides, clones, noms communs, modalité de production, d'extraction et de stockage...

- procédés d'industrialisation: principes et méthodes, procédés d'isolation des actifs, structure et propriétés chimiques, origine...

- informations biotechnologiques: origine biologique, activités enzymatiques, données séquentielles...

- composés exclus : mélanges intentionnels, les classes de substances, les organismes biologiques, les plantes entières, les noms de marque...

- L'INRS, Institut national de recherche de la sécurité, à rendu obligatoire avant toute mise sur le marché européen de toute substance chimique son enregistrement dans un inventaire européen des substances chimiques existantes :

- I'EINECS: European Inventory Of Existing Commercial Chemical Substances jusqu'en 1992, simple enregistrement,

- I'ELINCS : European List Of Notified Chemical Substances depuis 1992, enregistrement avec mise en place d'un dossier technique fonction des quantités mise sur le marché et de la présence éventuelle de polymères. Ce dossier comprend: des données physicochimiques relatives au nouvel ingrédient; des données techniques de production de ces ingrédients : une fiche de données de sécurité (tableau 3); des données d'innocuité et de sécurité qui seront d'autant plus importantes, que les tonnages mis sur le marché le seront. 


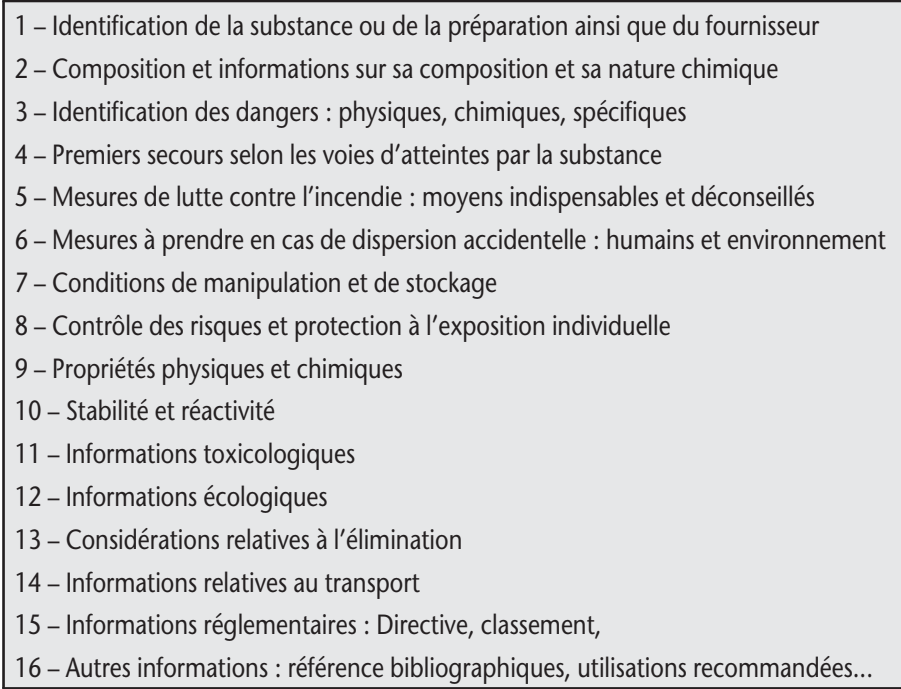

- INCI NAME : International Nomenclature Of Cosmetic Ingredients est la dénomination commune européenne des ingrédients cosmétiques mise en place par le COLIPA et reprise dans I'Inventaire Européen des Ingrédients Cosmétiques.

- De la même façon, la Cosmetic, Toiletries And Fragrance Association propose une nomenclature $I \mathrm{NCl}$ US des ingrédients cosmétiques admise aux États-Unis, avec publication dans : International Cosmetic Ingredient Dictionary and Handbook (dixième édition 2004).

\section{Les mentions obligatoires}

L'article 6 définit un certain nombre de mentions à faire figurer sur les emballages des produits cosmétiques : « Les produits cosmétiques ne peuvent être mis sur le marché que si le récipient et l'emballage portent en caractères indélébiles, facilement visibles et lisibles, un certain nombre de mentions... ».

En ce qui concerne les matières premières cosmétiques, doit figurer sur l'emballage le listing des ingrédients qui composent le produit, selon les règles suivantes :

- tous les ingrédients de la formulation ;

- liste des ingrédients dans l'ordre décroissant de leur importance pondérale au moment de leur incorporation jusqu'à $1 \%$;

- les ingrédients à concentration $<1 \%$ sont indiqués en ordre indifférent après les autres ingrédients ;

- en cas d'impossibilité pratique le symbole de la main sur le livre renvoie soit à une notice, soit à une étiquette, soit à tout autre support indicatif ;

- les ingrédients sont indiqués sur une face quelconque de l'emballage externe ;

- les dénominations $\mathrm{INCl}$ publiées dans l'inventaire cosmétique de la Commission européenne, LATINES selon la classification de LINNE pour les matières premières végétales (genre et espèce), les noms courants des Pharmacopées européennes et les numéros «Colour Index » $\mathrm{Cl}$ pour les colorants cosmétiques exception faite des colorants capillaires, PARFUM pour les ingrédients et compositions parfumantes et

- AROMA pour les ingrédients et compositions aromatisantes ;

- les colorants sont indiqués en ordres indifférents à la fin de l'énumération des autres ingrédients: [+/-] : notion de "peut contenir » prévu pour les colorants mais pouvant inclure $d^{\prime}$ autres substances entrant dans une gamme. Quand un colorant sera utilisé pour d'autres usages que la coloration le numéro $\mathrm{Cl}$ sera remplacé par la dénomination $\mathrm{INCl}$ : TITANIUM DIOXYDE et non Cl 77891 ;

- les mélanges d'ingrédients seront listés selon le pourcentage décroissant en matière active, solvants y compris ;

- les matières premières fournies intentionnellement en mélanges d'ingrédients, chacun des ingrédients doit être déclaré séparément en tenant compte de sa concentration dans le produit fini ;

- il est de la responsabilité du responsable sécurité produit de tenir compte de la présence des additifs ajoutés à des matières premières et de les inscrire ou de les dispenser d'étiquetage ; - les substances techniques nécessaires au process de fabrication qui conduisent chimique- ment à un autre composé offrent deux possibilités : STEARIC ACID/SODIUM HYDROXYDE ou SODIUM STEARATE ;

- substances revendiquées: aucune exigence de pourcentage quantitatif.

\section{Le dossier cosmétique}

Dans l'intérêt d'un traitement médical rapide et approprié I'article 7 définit un certain nombre d'informations adéquates et suffisantes, relatives aux produits commercialisés ainsi qu'aux substances utilisées dans les produits cosmétiques, qui pourront être tenues à la disposition des seules autorités compétentes, au travers de la mise en place d'un dossier cosmétique. Ce dernier devra contenir les informations suivantes :

- I'identité du fabricant ou de son mandataire, ou la personne pour le compte de qui le produit est fabriqué, ou le responsable de la mise sur le marché communautaire ;

- la formule qualitative et quantitative du produit en $\mathrm{INCl}$ de préférence ;

- les spécifications physicochimiques et microbiologiques des matières premières et des produits cosmétiques. Guidelines et normes ISO en cours ;

- la méthode de fabrication conformément aux bonnes pratiques de fabrication, la personne responsable de la fabrication ou de la première importation ayant des compétences reconnues;

- l'évaluation de la sécurité pour la santé humaine du produit fini selon les bonnes pratiques de laboratoire (texte en préparation), en prenant en compte le profil toxicologique général de chacun des ingrédients, leur structure chimique et leur niveau d'exposition (guidelines pour le safety assessment of cosmetic product) ;

- les noms et adresses des personnes qualifiées responsables du dossier d'évaluation de la sécurité du produit ;

- les données existantes en matière d'effets indésirables pour la santé humaine ;

- les preuves de l'effet revendiqué par le produit cosmétique lorsque la nature de l'effet ou du produit le justifie (guidelines for the evaluation of the efficacy of cosmetic products).

\section{Méthodes analytiques et bactériologiques}

L'article 8 stipule la nécessité de mentionner les méthodes d'analyse nécessaires au contrôle de la composition des produits cosmétiques ainsi que les critères de pureté microbiologique et chimique pour les produits cosmétiques accompagnés de leurs méthodologies. 


\section{Conclusion}

Les matières premières lipidiques entrant dans la composition de produits cosmétiques ne présentent pas de spécificités particulières et se doivent comme tout ingrédient cosmétique de répondre aux exigences de la Directive Cosmé- tique en vigueur dans le pays où ces derniers sont commercialisés.

La production et la commercialisation de matières premières lipidiques à des fins d'ingrédients cosmétiques, sont soumises aux réglementations en vigueur dans les pays de leur commercialisation.
II convient de suivre de près les travaux REACH de l'industrie chimique, quant aux matières premières cosmétiques lipidiques qui pourraient être mises en examen pour des compléments d'informations relatifs à la sécurité de leur utilisation. 